\title{
Studies of Quantum Mechanical Coherency Effects in Neutrino-Nucleus Elastic Scattering
}

\author{
Vivek Sharma ${ }^{a, *}$ and Henry T. Wong ${ }^{a}$ \\ ${ }^{a}$ Institute of Physics, Academia Sinica, \\ Taipei 11529, Taiwan \\ E-mail: vsharma@gate.sinica.edu.tw, htwong@gate.sinica.edu.tw
}

\begin{abstract}
Low energy neutrinos play important role in nuclear and astrophysical processes. Such neutrinos with energy below $\sim 100 \mathrm{MeV}$ are able to scatter off the nucleus via elastic scattering. Neutrinonucleus elastic scattering $\left(v A_{e l}\right)$ provides a unique laboratory to study the quantum mechanical coherency effects in electroweak interactions. We present the detailed study and formulation of coherency effects, relate this to nuclear form factors and experimental cross-section ratios. The parameters chosen to quantify the coherency are universally applicable to different neutrino sources and target nuclei. We characterize how the energy dependence of the coherence factor leads to complementary among measurements at various neutrino sources with different targets. We also provide the constraints on coherency for the first generation discovery measurements of $v A_{e l}$ with CsI target and theoretical expectations from Argon and Germanium target.
\end{abstract}

40th International Conference on High Energy physics - ICHEP2020

July 28 - August 6, 2020

Prague, Czech Republic (virtual meeting)

\footnotetext{
${ }^{*}$ Speaker
} 


\section{Introduction and Formulation}

Neutrino-nucleus elastic scattering $\left(v A_{e l}\right)$ is a well defined process in Standard Model (SM) of particle physics $[1,2]$ towards which several experimental programs are being pursued[3-8]. The $v A_{e l}$ is a flavor-blind process which is mediated by the exchange of $\mathrm{Z}$ boson. This makes the process a probe for the study of weak current in SM. The differential cross-section of $v A_{e l}$ scattering at three momentum transfer $q\left(\equiv|\vec{q}|\right.$ ) and incident neutrino energy $E_{v}$ on a target nuclei of mass $M$, can be expressed as[9]

$$
\left[\frac{d \sigma\left(q^{2}, E_{v}\right)}{d q^{2}}\right]_{v A_{e l}}=\frac{1}{2}\left[\frac{G_{F}^{2}}{4 \pi}\right] \cdot\left[1-\frac{q^{2}}{4 E_{v}^{2}}\right] \cdot \Gamma\left(q^{2}\right),
$$

where $G_{F}$ is fermi constant and term $\Gamma\left(q^{2}\right)$ describes the contribution of many-body physics of the target nuclei, since $v A_{e l}$ scattering involves collective contribution of individual nucleons in the nucleus.

The kinematics constraints on squared three momentum transfer $\left(q^{2} \simeq 2 M T\right)$ is $q_{\text {max }}^{2}=$ $4 E_{v}^{2}\left[M /\left(M+2 E_{v}\right)\right] \simeq 4 E_{v}^{2}$ and $q_{\text {min }}^{2} \simeq 2 M T_{\text {min }}$, where $T$ is the experimentally observable nuclear recoil energy expressed in $\mathrm{keV}_{n r}$ unit.

The formulation of $\Gamma\left(q^{2}\right)$ in eq. 1 depends on different aspects of physics probe. The usual description is based on nuclear physics given as

$$
\Gamma\left(q^{2}\right) \equiv \Gamma_{N P}\left(q^{2}\right)=\left[\varepsilon Z F_{Z}\left(q^{2}\right)-N F_{N}\left(q^{2}\right)\right],
$$

where $F_{Z}\left(q^{2}\right) \in[0,1]$ and $F_{N}\left(q^{2}\right) \in[0,1]$ are respectively, the proton and neutron form-factors, while $\varepsilon \equiv\left(1-4 \sin ^{2} \theta_{W}\right)=0.045$, gives the $\mathrm{N}^{2}$ enhancement to the cross-section. This description connects the $v A_{e l}$ scattering to nuclear physics. The proton form-factor gets contribution from electron nucleus scattering data, while neutron form-factor would require a probe from weak processes. Various formulation for nuclear form-factor are discussed in [10-12].

Another description of $\Gamma\left(q^{2}\right)$ comes from quantum mechanical coherency effects[13] given by

$$
\Gamma\left(q^{2}\right) \equiv \Gamma_{Q M}\left(q^{2}\right)=(\varepsilon Z-N)^{2} \cdot \alpha\left(q^{2}\right)+\left(\varepsilon^{2} Z+N\right)\left[1-\alpha\left(q^{2}\right)\right],
$$

where $\alpha\left(q^{2}\right) \equiv \cos \phi \in[0,1]$ is the degree of coherency given that $\phi$ is the misalignment phase angle. This description leads the limitation behavior of complete coherency state $\left(\alpha=1\right.$ at $\left.q^{2} \sim 0\right)$ with $\left(d \sigma / d q^{2}\right) \propto[\varepsilon Z-N]^{2}$ and decoherency state $\left(\alpha=0\right.$ at $\left.q^{2} \gtrsim[\pi / R]^{2}\right)$ with $\left(d \sigma / d q^{2}\right) \propto\left[\varepsilon^{2} Z+N\right]$.

An alternative measurement driven description is given by the cross-section reduction relative to complete coherency condition[13] as

$$
\Gamma\left(q^{2}\right) \equiv \Gamma_{D A T A}\left(q^{2}\right)=(\varepsilon Z-N)^{2} \cdot \xi\left(q^{2}\right),
$$

where the limiting behavior leads to complete coherency state at $\xi\left(q^{2}\right)=1$ and decoherency state at $\xi\left(q^{2}\right)=\left[\frac{\left(\varepsilon^{2} Z+N\right)}{(\varepsilon Z-N)^{2}}\right]$. The experimentally measurable cross-section suppression $\left(\xi\left(q^{2}\right)\right)$ is related to the quantum mechanical coherence and nuclear physics via, respectively,

$$
\xi\left(q^{2}\right)=\alpha\left(q^{2}\right)+\left[1-\alpha\left(q^{2}\right)\right]\left[\frac{\left(\varepsilon^{2} Z+N\right)}{(\varepsilon Z-N)^{2}}\right] \quad \text { and } \quad \xi\left(q^{2}\right)=\frac{\left[\varepsilon Z F_{Z}\left(q^{2}\right)-N F_{N}\left(q^{2}\right)\right]^{2}}{(\varepsilon Z-N)^{2}} .
$$


while both of these are connected by

$$
\left[\varepsilon Z F_{Z}\left(q^{2}\right)-N F_{N}\left(q^{2}\right)\right]^{2}=(\varepsilon Z-N)^{2} \cdot \alpha\left(q^{2}\right)+\left(\varepsilon^{2} Z+N\right) \cdot\left[1-\alpha\left(q^{2}\right)\right]
$$

The functions $\Gamma_{N P}\left(q^{2}\right), \Gamma_{Q M}\left(q^{2}\right)$ and $\Gamma_{D A T A}\left(q^{2}\right)$ can be directly measured from $v A_{e l}$ data. Prior to actual measurements, specific formulations of the nuclear form factors have to be adopted for phenomenological studies. One of the general description of the identical nuclear form factor for both proton and neutron $\left(F_{Z}\left(q^{2}\right)=F_{N}\left(q^{2}\right) \equiv F_{A}\left(q^{2}\right)\right)$ is given by Helm model, which can be expressed as[11, 13]:

$$
F_{A}\left(q^{2}\right)=\left[\frac{3}{q R_{0}}\right] j_{1}\left(q R_{0}\right) \exp \left[-\frac{1}{2} q^{2} s^{2}\right],
$$

where $j_{1}(x)$ is the first order spherical Bessel function and the target nuclei dependence is introduced through $R_{0}^{2}=R^{2}-5 s^{2}$, where $s=0.5 \mathrm{fm}$ and $R=1.2 A^{1 / 3}$. In this formulation the squared formfactor is equal to the cross-section suppression: $\left[F_{A}\left(q^{2}\right)\right]^{2}=\xi\left(q^{2}\right)$.
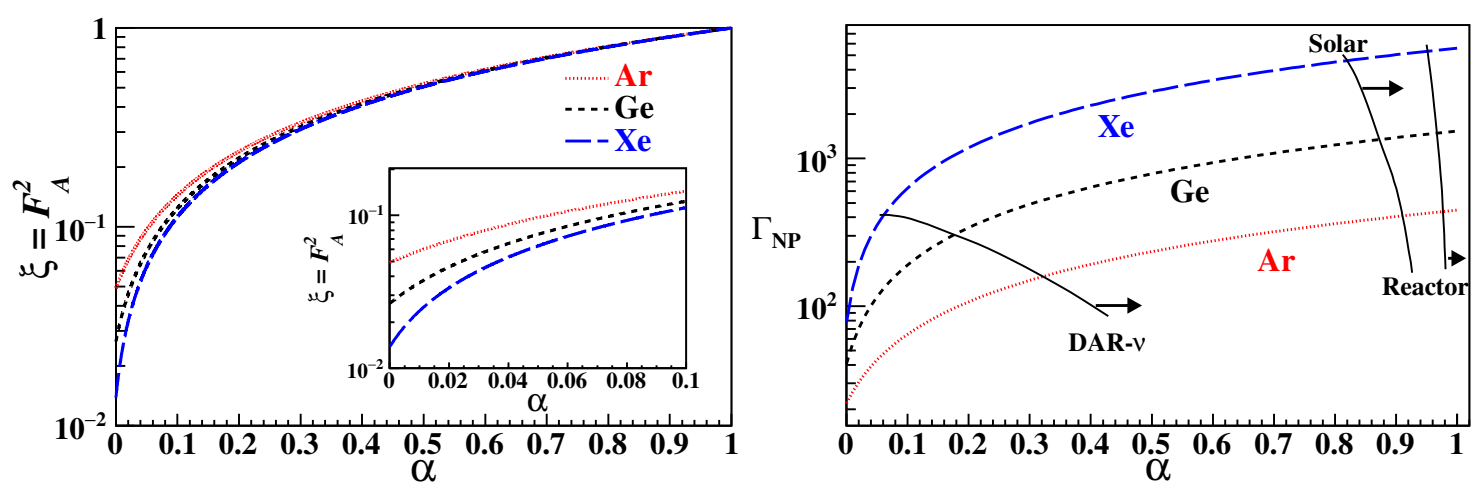

Figure 1: The variation of cross-section ratios $[\xi]$ (left) and many-body nuclear physics term $\left[\Gamma_{N P}\right]$ (right) with $\alpha$ for three target nuclei, independent of underlying nuclear physics.

The relation between $\xi$ and $\Gamma_{N P}$ with $\alpha$ is shown in Fig. 1 left $\&$ right for three representative nuclei. Contours of maximum- $q^{2}$ for different neutrino sources are marked in Fig. 1 right. The limiting domains corresponding to complete coherency and decoherency for $\Gamma_{N P}, \alpha$ and $\xi$ are summarized in Table 1. The relation $\Gamma_{N P}=\left(\varepsilon^{2} Z+N\right)$ for complete decoherency, is a result that emerges by relating $\Gamma_{N P}$ and $\Gamma_{Q M}$ in Eq. 6 , and could not be derived by considerations of nuclear form factor of Eq. 2 alone.

\section{Experimental Projection}

The $\Gamma\left(q^{2}\right)$ functions are directly measurable from $v A_{e l}$ data without the underlying physics input. Although, we need to choose the specific formulation of the nuclear form-factor for the phenomenological studies. Therefore, we used the frequently adopted nuclear form-factor formulation of Eq. 7. We studied the $v A_{e l}$ processes on several nuclei of experimental interest and at different mass ranges ( $\mathrm{Ar} ; \mathrm{Ge} ; \mathrm{Xe})$ with $\mathrm{Z}=(18 ; 32 ; 54)$ The first measurement of $v A_{e l}$ was done by CsI, having $\mathrm{Z}=55$ and 53 , respectively $[14,15]$. CsI is approximated as $\mathrm{Xe}(\mathrm{Z}=54)$ in this discussion. 
Table 1: The limiting cases of complete coherency and decoherency are described for three formulations of many-body physics in $v A_{e l}$ scattering.

\begin{tabular}{|ccc|}
\hline Conditions & Complete Coherency & Complete Decoherency \\
\hline \hline$q^{2}$ & $\rightarrow 0$ & $\gtrsim\left[\frac{\pi}{R}\right]^{2}$ \\
$\left(\right.$ I) $\Gamma_{N P}\left(q^{2}\right)=\left[\varepsilon Z F_{Z}\left(q^{2}\right)-N F_{N}\left(q^{2}\right)\right]^{2}$ & 1 & - \\
$F_{Z}\left(q^{2}\right)$ & 1 & - \\
$F_{N}\left(q^{2}\right)$ & $(\varepsilon Z-N)^{2}$ & $\left(\varepsilon^{2} Z+N\right)$ \\
$\Gamma_{N P}\left(q^{2}\right)$ & 0 & $\pi / 2$ \\
$\phi\left(q^{2}\right)$ & 1 & 0 \\
$\alpha\left(q^{2}\right)$ & & {$\left[\frac{\left(\varepsilon^{2} Z+N\right)}{(\varepsilon Z-N)^{2}}\right]$} \\
(II) $\Gamma_{Q M}\left(q^{2}\right)=(\varepsilon Z-N)^{2} \alpha\left(q^{2}\right)+\left(\varepsilon^{2} Z+N\right)\left[1-\alpha\left(q^{2}\right)\right]$ & 1 & $\propto\left(\varepsilon^{2} Z+N\right)$ \\
$($ III $) \Gamma_{D A T A}\left(q^{2}\right)=(\varepsilon Z-N)^{2} \xi\left(q^{2}\right)$ & $\propto(\varepsilon Z-N)^{2}$ & \\
$\xi\left(q^{2}\right)$ & & $\left.d \frac{d \sigma}{d q^{2}}\right]\left(q^{2}\right)$
\end{tabular}
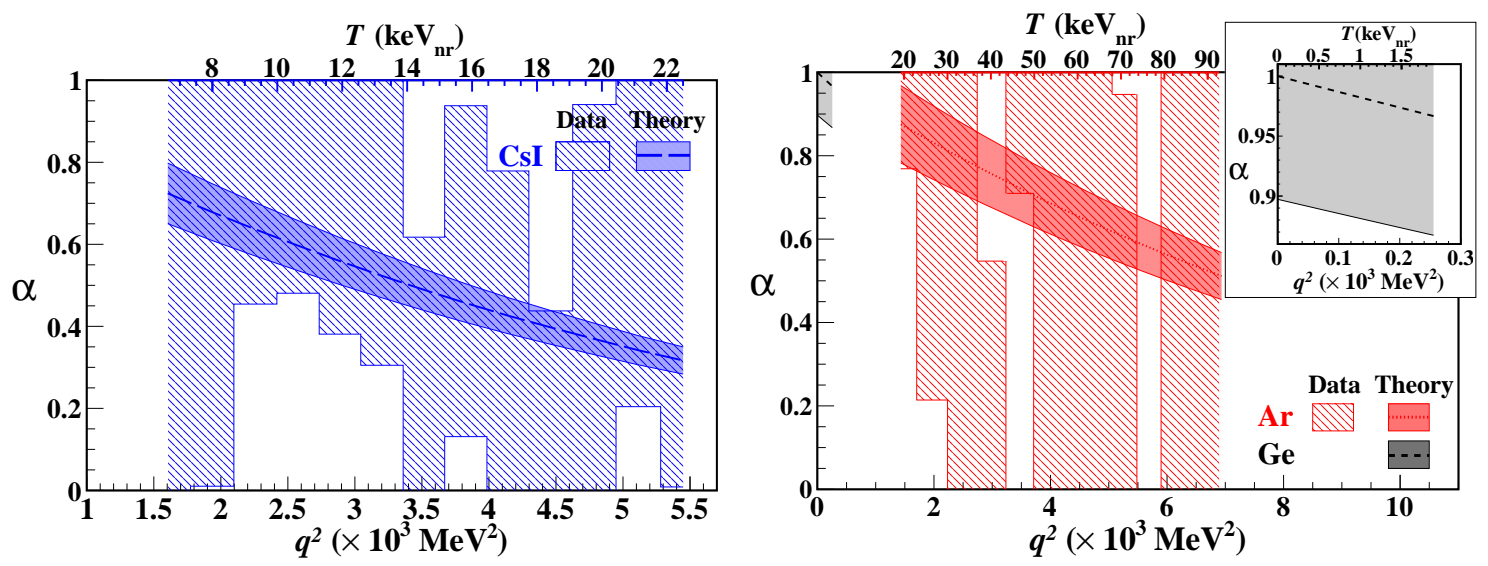

Figure 2: Measurements on $\alpha$ from COHERENT (left) CsI [15] and (right) Ar [16] data with DAR- $v$. The stripe-shaded areas are the 1- $\sigma$ allowed regions derived from the reduction in cross-section relative to the complete coherency conditions independent of nuclear physics input. The dark-shaded regions are the theoretical expectations adopting the nuclear form factor formulation of Eq. 9 with a $\pm 1 \sigma$ uncertainty of $10 \%$. The same projection applies to reactor- $v$ on Ge in (b) with $q^{2}$ range specified by FWHM in $\left[\Phi_{v} \cdot \sigma_{v A_{e l}}\right]$. The $\sigma\left(q^{2}\right)$ values for different nuclei can be consistently compared[9].

The bin-wise $\xi\left(q^{2}\right)$ cross-section suppression relative to the complete coherency condition was provided by measurements. The allowed 1- $\sigma$ ranges in $\alpha\left(q^{2}\right)$ are evaluated according to Eq. 7 and depicted as stripe-shaded region in Fig. 2. The results are data-driven without invoking nuclear physics input. The theoretical expectations adopting the nuclear form factor formulation of Eq. 9 with a $\pm 1 \sigma$ uncertainty of $10 \%$ are superimposed as dark-shaded bands, showing the cases with CsI (equivalently, Xe) and Ar at DAR- $\pi$, and with Ge at reactors.

The significance in terms of p-values of the CsI data in probing the specific cases of complete coherency and decoherency are excluded with $\mathrm{p}=0.023$ at $q^{2}=3.5 \times 10^{3} \mathrm{MeV}^{2}$ and with $\mathrm{p}=0.013$ 
at $q^{2}=2.6 \times 10^{3} \mathrm{MeV}^{2}$, respectively. The $90 \%$ confidence levels of these bounds are $\alpha(\phi)<0.73$ $(0.48 \pi / 2)$ and $\alpha(\phi)>0.37(0.76 \pi / 2)$, respectively.

These diverse ranges of $\alpha$-sensitivity indicate the complementarity of $v A_{e l}$ measurements among reactor and DAR- $\pi$-neutrinos. Future measurements of solar $v A_{e l}$ with multi-ton detectors would probe a similar range of $\alpha$ as reactor neutrinos. Xenon detectors with scale $O(100)$ ton would be required to probe the weakly coherent region at $\alpha<0.2$ with atmospheric neutrinos.

\section{Summary}

Elastic scattering of neutrino with nucleus provides a probe to study the quantum mechanical coherence effects in electroweak interactions. This interpretation of the process is complementary to the language of the nuclear form factors. We relate these approaches and provide constraints on degree of coherency $\alpha\left(q^{2}\right)$ on positive measurements of $v A_{e l}$. Current positive measurements on $v A_{e l}$ provide only weak constraints to $\alpha\left(q^{2}\right)$ and equivalently misalignment phase-angle $\phi\left(q^{2}\right)$. Data with $O 10 \%$ accuracy would allow the studies of coherency transitions.

We note that the interaction $v A_{e l}$ of Eq. 1 involves two distinct concepts: elastic kinematics and quantum mechanical coherency. The coherency aspect should be characterized by distributions with dependence on $A(Z, N)$ and $q^{2}$. Descriptions of coherency as a binary state or having both concepts bundled together may have the unintended consequences of missing the complexities of the process and suppressing the potential richness of its physics content.

The TEXONO experiment [17-20] is working on reactor $v A_{e l}$ with Germanium detector technology. The high energy resolution and low threshold germanium detectors have been used to study neutrino magnetic moment, neutrino millicharge and $\chi \mathrm{N}$ interactions[17, 21, 22]. The near future observation of $v A_{e l}$ with reactor neutrinos, can probe the mostly complete $(\alpha>95 \%)$ coherency region of $v A_{e l}$ scattering (inset of Fig. 2 right). Future measurements of solar $v A_{e l}$ with multi-ton detectors would probe a similar range of $\alpha$ as reactor neutrinos.

\section{References}

[1] D. Z. Freedman Coherent effects of a weak neutral current. Phys. Rev. D 9, 1389 (1974).

[2] D. Z. Freedman The Weak Neutral Current and its Effects in Stellar Collapse. Ann. Rev. of Nucl. Sci. Vol. 27, 167 (1977).

[3] H. T. Wong Research program towards observation of neutrino-nucleus coherent scattering. J. Phys. Conf. Ser. 39 (2006) 266-268 hep-ex/0511001.

[4] H. T. Wong Neutrino-nucleus coherent scattering and dark matter searches with sub-keV germanium detector. Nuclear Physics A 844, 229c-233c (2010).

[5] D. Akimov The COHERENT Experiment at the Spallation Neutron Source. ArXiv:1509.08702 (2016).

[6] G. Agnolet Background studies for the MINER Coherent Neutrino Scattering reactor experiment. Nucl. Inst. and Meth. in Phys. Res 853, 53-60 (2017). 
[7] A. Aguilar-Arevalo Results of the engineering run of the Coherent Neutrino Nucleus Interaction Experiment (CONNIE). JINST, 11, P07024 (2016).

[8] H. Bonet First constraints on elastic neutrino nucleus scattering in the fully coherent regime from the Conus experiment. ArXiv:2011.00210 (2020).

[9] V. Sharma Studies of Quantum-Mechanical Coherency Effects in Neutrino-Nucleus Elastic Scattering. ArXiv:2010.06810 (2020).

[10] G. Duda Model-independent form factors for spin-independent neutralino-nucleon scattering fromelastic electron scattering data. Journal of Cosmology and Astroparticle Phys., (2007).

[11] D. K. Papoulias Standard and Nonstandard Neutrino-Nucleus Reactions Cross Sections and Event Rates to Neutrino Detection Experiments. Adv. in High Energy Phys. 763648 (2015).

[12] M. Hoferichter Coherent elastic neutrino-nucleus scattering: EFT analysis and nuclear responses. ArXiv:2007.08529 (2020).

[13] S. Kerman et al. Coherency in neutrino-nucleus elastic scattering. Physical Review D 93, 113006 (2016).

[14] D. Akimov Observation of coherent elastic neutrino-nucleus scattering. Science 10.1126/science.aao0990, (2017).

[15] J. I. Collar Response of CsI[Na] to nuclear recoils: Impact on coherent elastic neutrinonucleus scattering. Phys. Rev. D 100, 033003 (2019).

[16] D. Akimov First Detection of Coherent Elastic Neutrino-Nucleus Scattering on Argon. ArXiv:2003.10630 (2020).

[17] H. T. Wong. Taiwan EXperiment On NeutrinO : History, Status and Prospects. The Universe 3 no. 4, 22-37 (2015), arXiv: 1608.00306.

[18] A. K. Soma et al. Characterization and Performance of Germanium Detectors with sub-keV Sensitivities for Neutrino and Dark Matter Experiments. Nucl. Inst. and Meth. in Phys. Res. A 836, 67 (2016).

[19] V. Sharma et al. Coherency in Neutrino-Nucleus Elastic Scattering. J. Phys.: Conf. Ser.1468 012149 (2020).

[20] V. Sharma et al. Status of the search of coherent neutrino nucleus elastic scattering at KSNL. Indian J Phys 92, 1145-1152 (2018).

[21] J. Chen et al. Atomic ionization of germanium by neutrinos from an ab initio approach. Phys. Lett. B 731, 159 (2014).

[22] J. Chen et al. Constraining neutrino electromagnetic properties by germanium detectors. Phys. Rev. D 91, 013005 (2015). 